\title{
Reliable Localization Systems including GNSS Bias Correction
}

\author{
Pierre Delmas, Christophe Debain \\ Cemagref \\ France \\ Roland Chapuis \\ LASMEA \\ France \\ Cédric Tessier \\ Effidence \\ France
}

\section{Introduction}

GNSS (Global Navigation Satellite System) system is the most famous system to realize localization of mobile robots. It is able to provide a global position everywhere in the world.

Moreover, as it provides a global position, it is not necessary to place the robot in known or structured environments (i.e. where several landmarks are available) to localize it unlike local positioning systems. So, the GNSS system has became an unavoidable system to guide automatically mobile robots.

Generally, GPS-RTK (Real Time Kinematic) sensors are used to localize and guide automatically mobile robots in outdoor environment. This type of sensor has a precision about few centimeters. However, they are expensive and difficult to use because they need differential correction to improve their precision. Consequently, an other GPS receiver is necessary as a reference. Moreover, their accurate measure is not always available because of GPS signal losses or multipaths. In this case, their accuracy to within 2 centimeters which is their main advantage, is not always available. So, it is impossible to use GPS-RTK sensors alone to have an effective localization system in the context of autonomous guidance. It must be used with other sensors to insure the localization when GPS signal is not available (Pein et al., 2006).

Some research makes an effective localization system for mobile robots using GPS-RTK with other satellite sensors. However, the dissemination of automatic guidance system in outdoor environment can use a low-cost sensors as natural GPS or GPS with a differential correction (WAAS or EGNOS). This type of sensors are less expensive than GPS-RTK and easy to use, because the operator has just to use and manage a single receiver. This last one has an accuracy between 1 and 3 meters with WAAS or EGNOS differential correction. To enhance their precision, natural GPS data can be fused with other exteroceptive data as it is done in (Tessier et al., 2006a). Generally, these localization systems are based on a Kalman Filter (KF) because 
autonomous guidance systems use a mono hypothesis localization estimation.

Nevertheless, there is an important assumption to use a KF, the errors of each sensor must be zero-mean, Gaussian and white process. If this assumption is not true, the estimation of position will be biased. Indeed, for every new observations of sensors data, the precision of localization (i.e. size of confidence ellipsis built with the covariance matrix) increases but the estimated position diverges from the true position (figure 1).

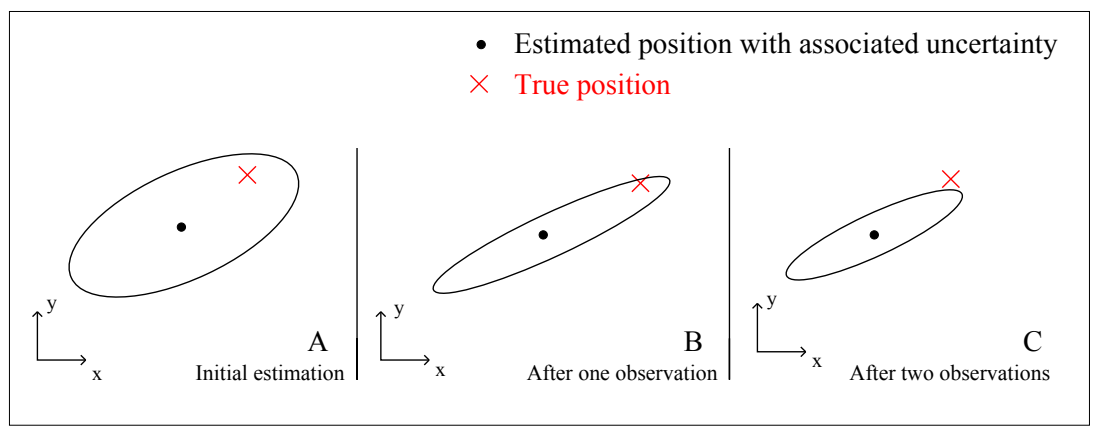

Fig. 1. Localization results after the fusion of two observations coming from a colored process (A at $t_{0} ; \mathrm{B}$ at $t_{1} ; \mathrm{C}$ at $t_{2}$ with $t_{0}<t_{1}<t_{2}$ )

In this figure, the situation $\mathrm{C}$ shows the true position is out of estimated position (position with its associated uncertainty). So, it becomes impossible to check the reliability of the localization. Consequently, the estimated position becomes useless, the mobile robot doesn't know where it is truly and it is impossible to accurately guide the vehicle. This is critical and dangerous situation which must be imperatively avoided. This critical situation appears for the localization systems which use GNSS receiver because generally they don't take care of the GNSS error characteristics. So, we propose in this paper a method to improve GNSS receiver precision and accuracy in the context of autonomous guidance.

In section II, we suggest an analysis of GNSS bias characterisation, one process to establish a model of it, and a solution to detect disturbances in GNSS data. Then, in the section III, we show how to improve easily a KF by inserting the prediction model and the condition. We will see the result of this method showing the robustness of GNSS bias modeling and the improvement of our localization system in the last section.

\section{Characterisation of the GNSS error}

The observations of GNSS data cause an unreliable estimated position. To better understand the problem, we propose an analysis of GNSS data.

\subsection{Data Analysis from a GNSS receiver}

The GNSS systems are based on localization by triangulation. The satellites send a message with the information (time and satellite ephemeris) allowing the receivers on the Earth calculate their position. Unfortunately, the GNSS system is not perfect. Many measurement errors cause a bad localization like satellite clock errors, ephemeris errors, atmospheric (ionosphere 
and troposphere) errors, multipath effects, receiver noise and resolution (for more information see (Kaplan, 1996)). Table 1 summarizes these errors with their characteristics.

\begin{tabular}{|c|c|c|}
\hline Error sources & Precision & Errors Characteristic \\
\hline Satellites clock errors & \pm 2 meters & stable \\
\hline Satellites ephemeris errors & \pm 2.5 meters & stable \\
\hline Ionospheric effects & \pm 5 meters & stable \\
\hline Tropospheric effects & \pm 0.5 meters & stable \\
\hline Multipath effects & \pm 1 meter & unpredictable \\
\hline Receiver noise and resolution & \pm 1 meter & unpredictable \\
\hline
\end{tabular}

Table 1. GNSS Error Sources.

We see that most of error sources are stable process (i.e. they evolve slowly). If we compare the low-cost GPS data with the true position coming from a GPS-RTK receiver (figure 2), we see that the natural GPS error seems to be constant. If we observe the auto-correlation of GPS longitude error between each iteration in static condition (figure 3), we realize this error is very correlated between successive iterations (it is the same case for GPS latitude error). It means the GPS error is a non white process. It is commonly named the GPS bias.

If we estimate the position with only the GNSS observations (position and associated covariance given by GNSS receiver) thanks to a KF, we obtain very quickly an unreliable estimated position (figure 4). It is always possible to increase the covariance of GNSS data. However, although this solution increases the reliability of GNSS measurement, the real problem is the GNSS error is a stochastical process. As soon as the position is calculated by KF thanks to GNSS observations, the estimated uncertainty decreases too much and consequently, the system becomes unreliable. So, it is necessary to know the value of GNSS error every time so as to have a reliable estimated position.

\subsection{GNSS error modelizing}

We have seen the main problem of inefficiency of localization system based on a KF is the non white GNSS error. So, we must find a model which describes this bias.

Some researcher proposed to find global criteria determining GNSS error (Nienaber \& Diekhans, 2006). These criteria consider only mean and standard deviation of the error in static and dynamic condition during 24 hours. However, many applications (agricultural tasks, automatic guidance, ...) run usually several hours and the characteristics (means and variance) of GNSS error are not the same for 24 hours. Table 2 represents the mean and standard deviation of data for three different moments. So, it is impossible to determine a reliable model with only mean and variance of GNSS error.

\begin{tabular}{|c|c|c|c|c|}
\hline Database & \multicolumn{2}{|c|}{ Longitude (m) } & \multicolumn{2}{c|}{ Latitude (m) } \\
& mean & RMS & mean & RMS \\
\hline Database 1 (2 hours) & 1.05 & 1.27 & 0.24 & 1.81 \\
\hline Database 2 (2 hours) & 2.72 & 1.31 & -1.62 & 1.24 \\
\hline Database 3 (2 hours) & 1.46 & 2.03 & -0.70 & 1.26 \\
\hline
\end{tabular}

Table 2. Means and Variances of data at three different moments.

Another solution is to estimate the GNSS error by inserting it in the state vector of KF as it is described in (Perera et al., 2006). A method to determine bias sensor online is proposed. The 


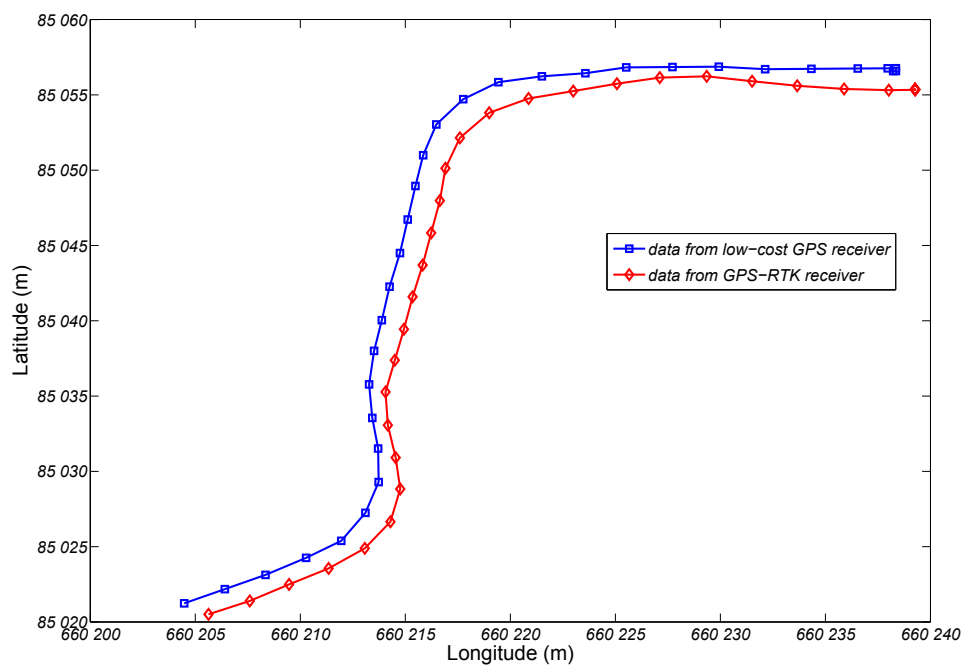

Fig. 2. Example of GPS data coming from low-cost GPS (blue) and RTK-GPS (red)

error is determined thanks to an exteroceptive sensor. We propose to improve this solution inserting a more accurate prediction model for GNSS error.

We make the assumption that the GNSS data is composed by position $(\mathrm{x}, \mathrm{y})$, an stochastical process $\left(\left(b_{x}, b_{y}\right)\right.$ the associated GNSS bias) and a zero-mean, white, Gaussian noise $\left(\epsilon_{x}, \epsilon_{y}\right)$ like (1). So, the aim is to look for a model which is able to predict the GNSS bias. This model must be determined to have a residual error which is zero-mean, white, Gaussian process. Then, this bias prediction model will be inserted in the KF to determine it and the position in the same time (see section III). The observation error of KF becomes zero-mean, white, Gaussian and doesn't drift the localization.

$$
\text { Data }_{\text {gnss }}=\left(\begin{array}{l}
x \\
y
\end{array}\right)+\left(\begin{array}{l}
b_{x} \\
b_{y}
\end{array}\right)+\left(\begin{array}{l}
\epsilon_{x} \\
\epsilon_{y}
\end{array}\right)
$$

We have seen GNSS bias is a stochastical model. To answer the problematic, we choose to use an AutoRegressive process described in (Laneurit et al., 2006) by Laneurit. Indeed, the AR process is a filter which has a zero-mean, white, Gaussian process in input and the stochastical process to determine in output. It is often used for a vocal identification like in (Kotnik \& Kačič, 2007). It is formulated in Z-transform by (2).

$$
F_{A R}=\frac{Y(z)}{\epsilon(z)}=\frac{1}{1+\alpha_{1} z^{-1}+\alpha_{2} z^{-2}+\ldots+\alpha_{p} z^{-p}}
$$

with $Y$ the stochastical signal, $\epsilon$ the zero-mean, white, Gaussian process, $\alpha$ and $p$ respectively the parameters and the order of AR process. In our case, the expression (2) becomes (3) in discrete domain. 


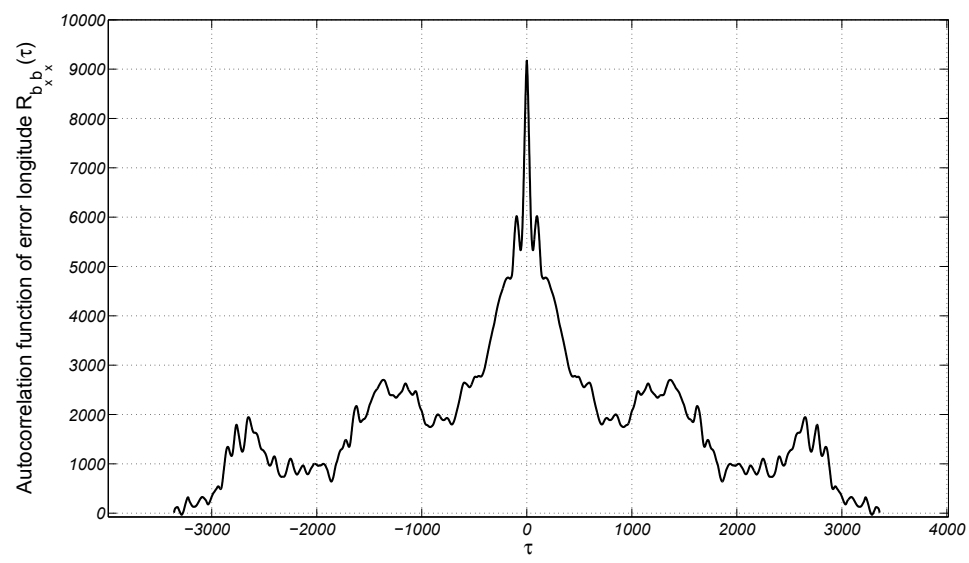

Fig. 3. Autocorrelation function of longitude error

$$
b_{k+1}=\sum_{i=1}^{p}-\alpha_{i} \cdot b_{k-i+1}+\epsilon_{k}, k \in[1: N-1], p \in \aleph^{*}
$$

with $b_{k}$ the GNSS bias at $k^{\text {th }}$ iteration. Now, it must find the parameters and the order of the AR process. This determination is made thanks to preliminary database of GNSS receiver. To always have a stationary model, the AR parameters are calculated by Burg method (Burg, 1975). Now, for the choice of AR process order, we take the order at the point where the power of $\epsilon$ stops decreasing significantly. The figure 5 represents the power of residual error between the real and estimated GNSS bias value for different AR order. Indeed, if AR process order is too reduced, the process won't represent the intrinsic properties due to GNSS signal. However, if AR process order is too big, the process will represent the properties due to signal noise. Other criteria exist as AIC (Akaike Information Criteria) and BIC (Bayesian Information Criteria) (Akaike, 1973) and (Schwarz, n.d.).

We have established the prediction model of GNSS bias thanks to AR process. But, the losses of one satellite may cause a disruption of GNSS observation. The figure 6 shows the GNSS observations change abruptly (about 50 centimeters) at time $t=6160$ s. Another important example of data disturbances is the multipath effects. When the receiver is close to an obstacle, the GNSS signal may reflect on this obstacle before to be received. This quick evolution causes disturbances on the prediction model. Consequently, to always have a GNSS localization reliable, these phenomena must be detected so as to reset the GNSS bias estimation.

The proposed idea to solve this problem is to compare the predicted GNSS data with the observation thanks to the Mahalanobis distance in (Laneurit, 2006). Generally, this distance is used to detect spurious data like in (Wu \& Zhang, 2006). Contrary to Euclidean distance, it takes care of data correlation. It is formalized by (4).

$$
d=\sqrt{\left(Z_{g n s s, k}-\hat{Z}_{k}\right)^{T} \cdot\left(\hat{R}_{k}+R_{g n s s}\right)^{-1} \cdot\left(Z_{g n s s, k}-\hat{Z}_{k}\right)}
$$




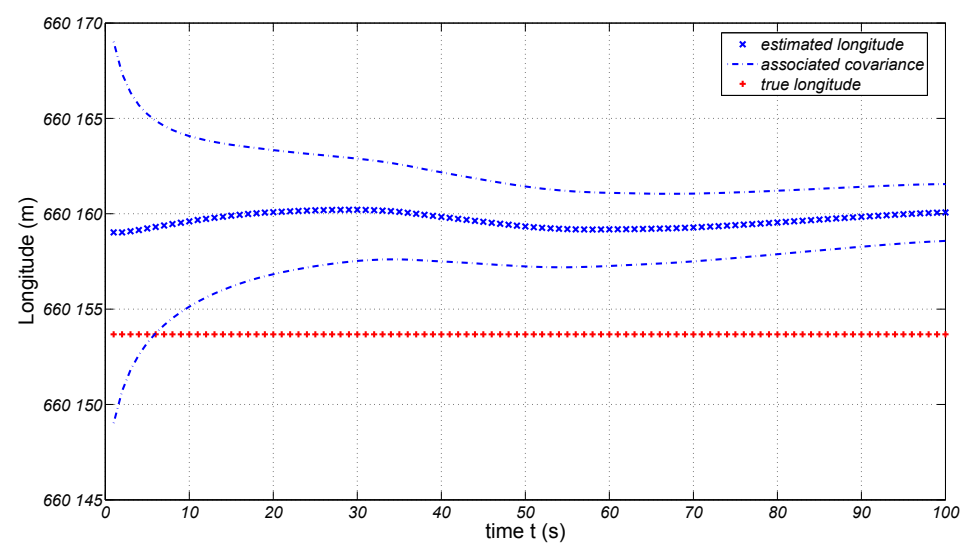

Fig. 4. Longitude estimation by KF with only GNSS observations with variance given by GNSS receiver (at $t=5$, the estimated position becomes false)

with $Z_{g n s, k}$ the GNSS observation in time $k$, the matrix $R_{g n s s}$ is the covariance matrix of the GNSS observation, $\hat{Z}$ and $\hat{R}$ are respectively the predicted value of GNSS data and its associated covariance. If the Mahalanobis distance between predicted and observed GNSS data is bigger than three, the GNSS localization system must be reset. Now, we are able to predict the stochastical GNSS error so as to determine it with the position thanks to KF. That leads to have only an observation error which can be considered like zero-mean, white, Gaussian noise. We have all information to create an localization system reliable using GNSS system.

\section{Integration of GNSS error model in KF}

In the previous part, we have established the prediction model of GNSS bias for latitude and longitude. Now, we will see how it is inserted in the KF. For GNSS localization, the state vector of KF is $X$ defined by (5).

$$
X_{k}=\left(x_{k}, y_{k}, b_{x, k}, \ldots, b_{x, k-p_{x}-1}, b_{y, k}, \ldots, b_{y, k-p_{y}-1}\right)^{T}
$$

with $x_{k}, y_{k}$ the estimation of Cartesian coordinates, $b_{x, k}, b_{y, k}$ bias of respectively longitude and latitude at $k^{\text {th }}$ iteration and $p_{x}$ and $p_{y}$ the prediction model order of respectively for the longitude and the latitude model. The choice of AR process order for the prediction bias model is very important because it determines the size of state vector of KF (in this case, $\operatorname{Size}(X)=2+p_{x}+p_{y}$ ). If the size of state vector is too big, the computing time will be too long for real-time applications and the automatic guidance can lost its stability.

The Kalman filter is composed in three parts : initialization, Prediction level and Correction Level. We will describe each part for the bias estimation and add a new part for the detection of disruption GNSS data. 


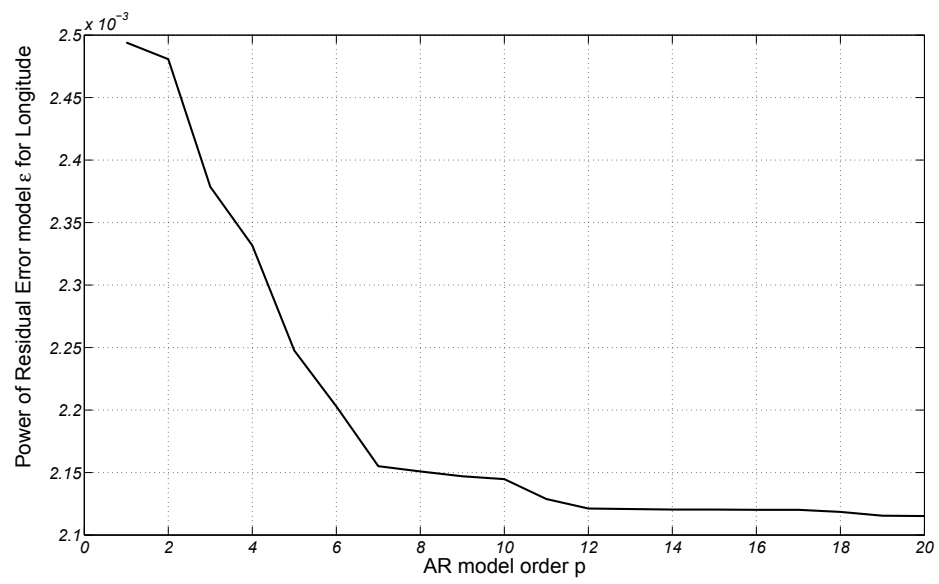

Fig. 5. Power of error predicted model $\epsilon(p)$ for longitude. The best order is 8 .

Initialization : Generally, the GNSS bias and the position are unknown. In that case, it is impossible to determine them. Indeed, the GNSS observation represents the sum of them and it is impossible with only GNSS data to separate them. We have an observation problem. We are obliged to know one of them to begin the estimation. To show the precision and the reliability of our method, we assume to begin the estimation with the known position $(5 \mathrm{~cm}$ precision) and the bias with a precision of $10 \mathrm{~m}$. In our localization systems, we use data coming from exteroceptive sensors. Then, the system can localize it without this information until the new reset.

Prediction Level : In the previous section, we have seen equation of bias evolution (3). This equation is the prediction equation of GNSS bias for the KF. So, the state evolution matrix $A$ is composed of three submatrices: $A_{x, y}$ for Cartesian position; $A_{b_{x}}$ and $A_{b_{x}}$ for respectively the longitude and the latitude GNSS bias. The AR parameters are easily inserted in the submatrix $A_{b_{x}}$ for example like (6).

$$
A=\left(\begin{array}{ccc}
A_{x, y} & 0 & 0 \\
0 & A_{b_{x}} & 0 \\
0 & 0 & A_{b_{y}}
\end{array}\right) \text { with } A_{b_{x}}=\left(\begin{array}{cccc}
-\alpha_{1} & \cdots & \cdots & -\alpha_{p_{x}} \\
1 & \cdots & 0 & 0 \\
\vdots & \ddots & \vdots & \vdots \\
0 & \cdots & 1 & 0
\end{array}\right)
$$

The variance of prediction equation $\epsilon$ is inserted in the prediction covariance matrix $Q$.

Detection of GNSS data disturbances : In the previous part, we have established a condition to detect disturbances of GNSS data. This condition is based on the Mahalanobis distance. This detection must be before the correction level to not degrade the estimation and after the prediction level of KF to allow the comparison between predicted and observed GNSS data. If the condition is respected, the estimation continues else the estimation must be initialized. 


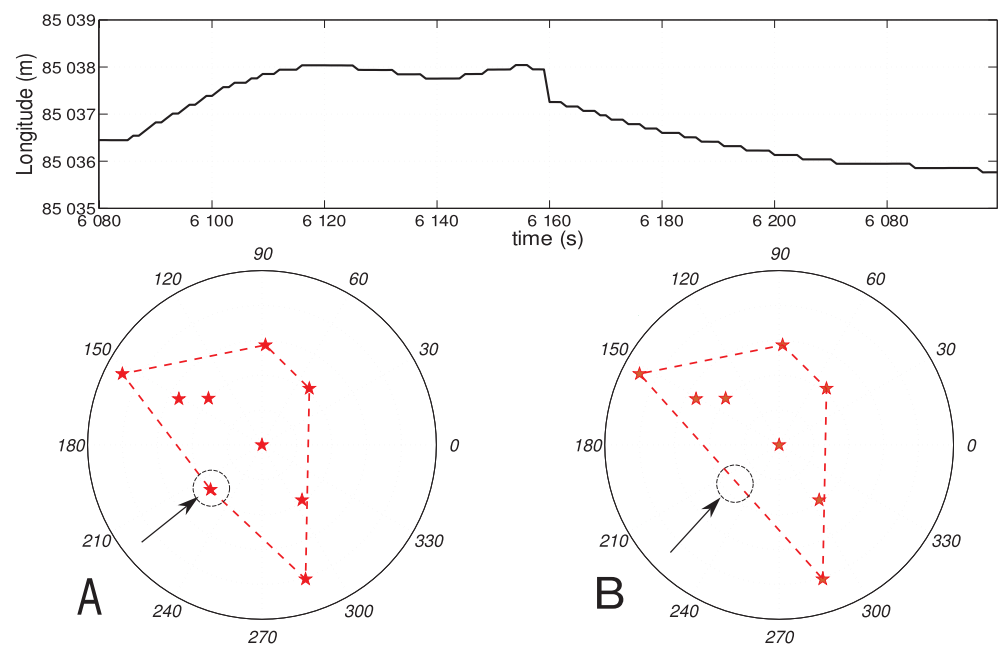

Fig. 6. Constellation influence on GNSS data. The situation A is the satellite constellation at $\mathrm{t}=6159 \mathrm{~s}$ and the situation $\mathrm{B}$ at $\mathrm{t}=6160 \mathrm{~s}$. Between the two situations, the pointed satellite disappears.

Correction Level : Now, the observed data is not only composed by the position but it is the sum of the position and the associated bias. We have seen in the initialization, that causes problem of observability. But, we make assumption the position is known with other sensor in the initialization. The state observation matrix for the GNSS $H_{g n s s}$ is (7).

$$
Z_{g n s s, k}=H_{g n s s} * X \text { with } H_{g n s s}=\left(\begin{array}{lllllll}
1 & 0 & 1 & 0 & 0 & \ldots & 0 \\
0 & 1 & 0 & 1 & 0 & \ldots & 0
\end{array}\right)
$$

To determine the power of their noise, we have made assumption that position data and bias are signal which are only composed of low-frequency component so we have filtered data signal by a high-pass filter and we have estimated the power of residual signal is equal to those of observation error. This power is integrated in observation covariance matrix $R_{g n s s}$. We see we have an observability problem. To solve this problem, we must know at a moment the position or the bias. In the initialization of localization systems, we know the true position thanks to GPS-RTK. It is possible to determine position by exteroceptive sensors by example. To resume, we obtain the figure 7 .

\section{Results}

In this part, we present results of localization using GNSS with bias correction as we propose in section III. First, we will see the localization results with only GNSS receiver. Then, we will present our localization system for autonomous guidance and the improvement of this method. 


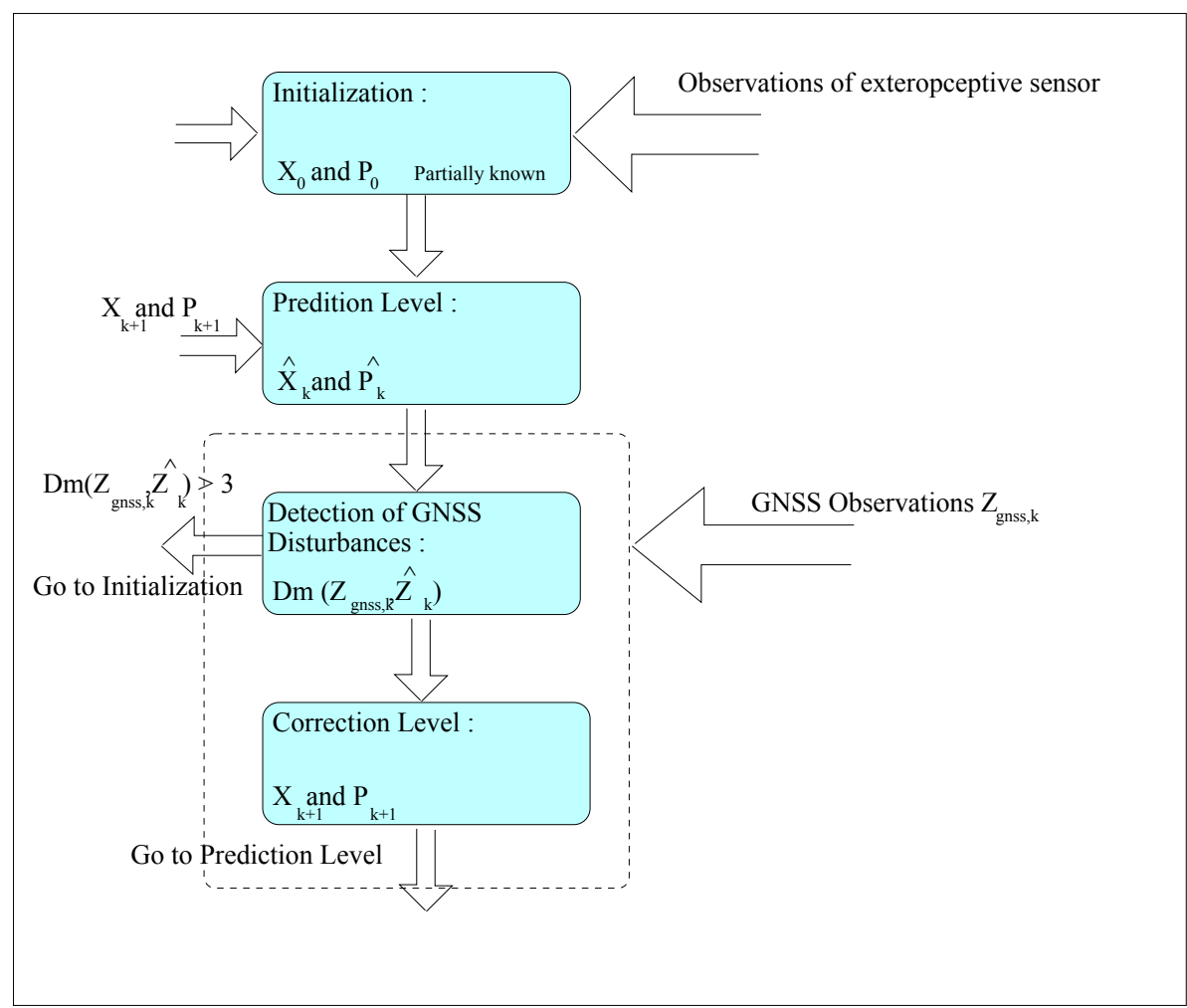

Fig. 7. Principle of Kalman filter with the detection of GNSS disturbances

Localization with GNSS alone : This test is done with a low-cost GPS receiver Holux GR213. We have established the prediction model in off-line before the test. Then, we have made the test in static condition during two hours for different orders of AR process without the condition to detect GNSS disturbances. The purpose of this test is to show the improvement of precision and the reliability of the method for a long period. Table 3 summarizes the results. In this table, we see that the choice of AR order is not an important parameter as it is shown by the little difference between each model. We can use the AR1 process which is the easiest model and the best for computing time. The figure 8 represents the estimated longitude with our method. We see the estimation is reliable for a long time in spite of GNSS disturbances. This result shows the robustness of prediction model. So for the second test, based on the evaluation of the quality of a localization process using a low cost GNSS receiver, we will use the AR1 process to establish a model of the GNSS stochastical error.

Localization for autonomous guidance : We have inserted the bias modeling in our localization system used to automatically guide our small mobile robot AROCO (see figure11). The sensors on-board the vehicle, amongst others, include a fiber-optic gyroscope (FOG) [KVH DSP 3000], rear and front wheel encoders, a low-cost GPS ( $5 m$ accuracy), a SICK PLS200 laser measurement system and a CCD camera (SONY VL500). The SICK PLS200 provides range 


\begin{tabular}{|l|c|c|c|c|c|c|}
\hline Bias model & \multicolumn{3}{|c|}{ Error for longitude (m) } & \multicolumn{3}{|c|}{ Error for latitude (m) } \\
& mean & max & RMS & mean & max & RMS \\
\hline $\begin{array}{l}\text { None (variance of GNSS } \\
\text { error given by the re- } \\
\text { ceiver) }\end{array}$ & 1.31 & 3.33 & 0.53 & -0.91 & 1.42 & 0.34 \\
\hline AR 1 Process & & & & & \\
\hline AR 2 Process & -0.015 & 0.040 & 0.009 & -0.010 & 0.045 & 0.011 \\
\hline AR 3 Process & -0.009 & 0.249 & 0.066 & -0.009 & 0.29 & 0.078 \\
\hline AR 4 Process & -0.022 & 0.197 & 0.081 & -0.147 & 0.356 & 0.094 \\
\hline AR 5 Process & 0.007 & 0.217 & 0.098 & -0.194 & 0.424 & 0.122 \\
\hline
\end{tabular}

Table 3. Error for Estimated position in static condition.

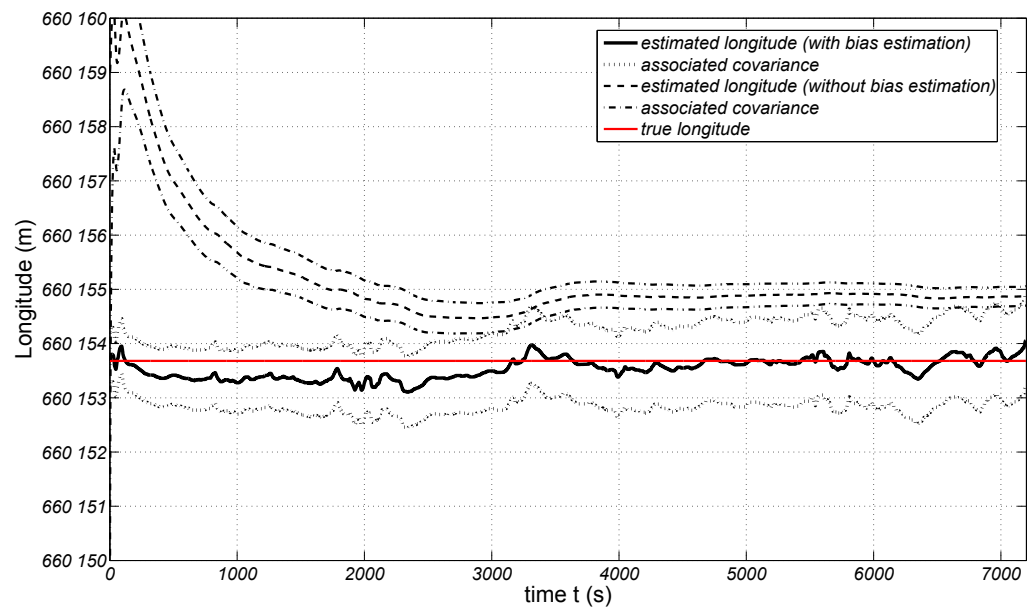

Fig. 8. Longitude estimation by KF taking care of GNSS stochastical error

measurements to object ahead at $0.5^{\circ}$ intervals over a span of $180^{\circ}$ in one scan. The scanned data arrive every 80ms. The SONY VL500 gives 7.5 640x480 YUV422 images per second. Finally, the robot is equipped with two on-board computer systems. The first running Linux RTAI is a low level system responsible for driving engine. The second running Linux is a high level system where our localization algorithm runs and sends control commands to the first PC.

Our localization system (developed by Tessier (Tessier et al., 2006a)) fuses local information with cheap GPS data (Tessier et al., 2006b). It estimates the vehicle's state (absolute position and orientation with their incertitude and their reliability) in a world reference frame represented by dynamic GIS (Geographic Information System). Local information given by landmarks detection allows the system to improve the position of localization given by natural GPS. Thanks to the use of proprioceptive and local information, an automatic guidance system can estimate the reliability of its localization (Tessier, Berducat, Chapuis \& Bonnet, 2007). Before the correction of the GNSS bias, the only way to use natural GPS in our guidance sys- 


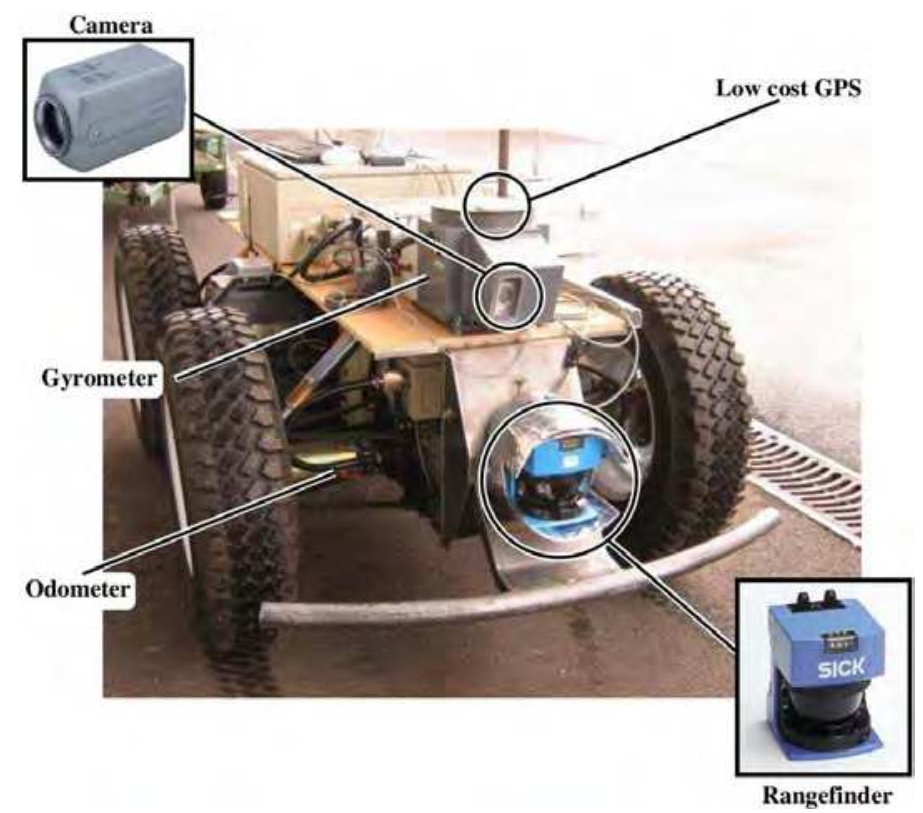

Fig. 9. Mobile robot used in experiments.

tem with an admissible level of reliability was to increase the GPS given by receiver. With our correction of GPS bias, the precision and the reliability of natural GPS are enough high to increase the performance of the guidance process.

Numerous real experimentations were made in outdoor environment to measure system capabilities and to attest the efficiency of our approach. We test it on our vehicle along the trajectory presented in figure (figure 11). Due to the presence of trees along the trajectory, we have satellite losses and lots of changes of satellite constellation.Thus, the vehicle moved automatically in wooded environments, in clear area, close to buildings, in hilly ground and under difficult climatic conditions (i.e. sunny weather, hailstorm, ...). The length of trajectory is about 400 meters and the speed of the vehicle is maintained at approximatively $3 \mathrm{~m} / \mathrm{s}$. The purpose of GPS bias estimation is to improve precision and particularly the reliability of our localization system. In our experimentation, we observe the estimated position of vehicle is more reliable than the past experimentation (the detection of landmarks is always successful contrary to the past experimentation). However, the localization system must be sometimes initialised because of GNSS disturbances and must use exteroceptive sensor (every 1 minute approximatively). If the landmarks is not available for a long time, the localization system doesn't certify its reliability, however the drift of estimated position due to GNSS disturbances is very small in comparison at the past.

- For this experiment, the vehicle succeeds in tracking the pre-defined trajectory with a good accuracy. However to check the repetitiveness and the sturdiness of the proposed approach, the vehicle reproduces faithfully the trajectory during 10 laps without stopping. Seven checkpoints have been placed on the path to measure the positioning errors. Those errors are presented in Table 4. They corresponds to the real lateral deviation of the vehicle with the reference path during the autonomous driving (i.e. localization and guidance). As we can notice, 


\begin{tabular}{|c|c|c|c|c|c|c|c|}
\hline & A & B & C & D & E & F & G \\
\hline mean $(\mathrm{cm})$ & -1.7 & -0.2 & 0.5 & -0.2 & 0 & -0.7 & -0.1 \\
\hline std $(\mathrm{cm})$ & 4.7 & 3.2 & 3.7 & 3.1 & 3.9 & 1.4 & 2.2 \\
\hline max $(\mathrm{cm})$ & 9.2 & 6 & 7.3 & 4.5 & 6 & 3.6 & 5.2 \\
\hline
\end{tabular}

Table 4. Real lateral deviation during path following for 10 laps.

the system is very accurate, the max error is below $10 \mathrm{~cm}$. The use of a multi-sensor system with an active search approach permits to locate the vehicle accurately with real-time constraints.

- Even in presence of disturbances, the system behaves correctly. The day of this experimentation, a strong downpour of hail fell. This disturbed the landmarks detection. Indeed, some hailstones are detected by the range-finder (figure 10). Thanks to the focusing process, this problem is attenuated and permits to identify some landmarks. Nevertheless, the system failed in detecting other landmarks because they are masked by the hailstones. Consequently, the reliability decreases and the vehicle speed slackens off. However, the system searches more easily recognizable landmarks (lane side with the camera, wall with the range-finder) to strengthen the estimation (i.e. increase the reliability) and boost the speed. At the end, the SLAG approach is robust.
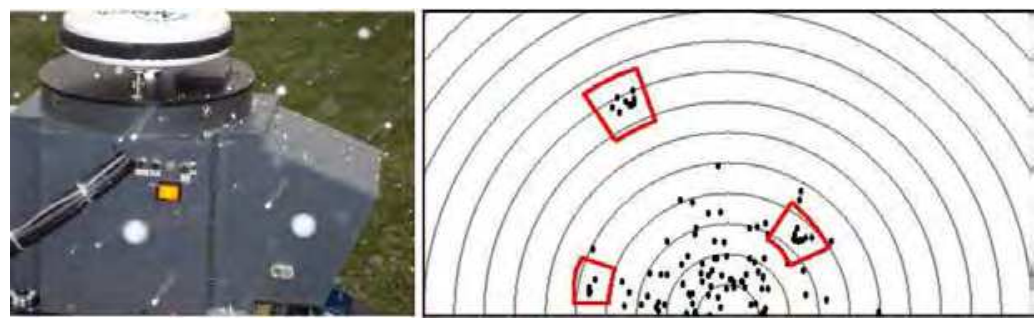

Fig. 10. (left) A strong hailstorm during our experimentation.

(right) Range-finder measurement with three trees and their region of interest.

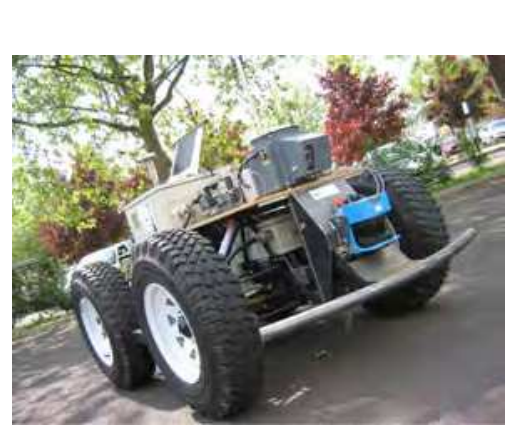

(a) The vehicle Aroco

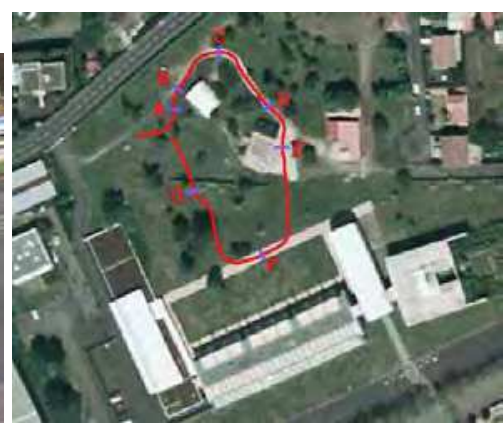

(b) Trajectory for the test

Fig. 11. Trajectory for the test with vehicle Aroco 


\section{Conclusion}

In this chapter, we have presented a method to improve localization systems based to data association with GNSS receiver. This method increases the precision and the reliability of localization based on an Kalman filter. It consists to take care the characteristics of GNSS error. This error is an unpredictive stochastic process and it drifts the estimated position which is calculated by a Kalman Filter. The developed idea is to establish a prediction model of GNSS bias and to insert it in the localization system so as to modify the observation error from low-cost GNSS receiver to zero-mean, white, Gaussian noise. We have seen a possible model of GNSS error is Autoregressive process. We have determined its parameters and its order. Then, we have shown how this model is inserted in the Kalman Filter. However, the bias estimation needs to have sometimes absolute data (position of landmark of the environment) coming from exteroceptive sensors. To do that we propose to use a multi-sensor system (Tessier, Debain, Chapuis \& Chausse, 2007) in which landmarks detection is given by autonomous entities called "perceptive agents". The main weakness of this multi-agent fusion system is about the focusing process and the measure of the accuracy of the estimated vehicle's pose. Thanks to numerous experiments we noticed a strong robustness and a good accuracy of the guidance process allowing using it at high speed even in an environment with lots of elements like trees or buildings.

\section{References}

Akaike, H. (1973). Information theory and an extension of the maximum likelihood principle, Proceeding of the $2^{\text {nd }}$ International Symposium on Information Theory, Budapest, pp. 267281.

Burg, J. (1975). Maximun entropy spectral analysis, Departement de Geophysiques, Universite de Stanford, Stanford, Californie, USA .

Kaplan, E. (1996). Understanding GPS: Principles and Applications, Artech House.

Kotnik, B. \& Kačič, Z. (2007). A noise robust feature extraction algorithm using joint wavelet packet subband decomposition and ar modeling of speech signals, Signal Process Vol.87: 1202-1223.

Laneurit, J. (2006). Perception multisensorielle pour la localisation de robot mobile en environnement extérieur, Phd thesis, Universite Blaise Pascal-Clermont-Ferrand, France .

Laneurit, J., Chapuis, R. \& Chausse, F. (2006). Accurate vehicle positioning on a numerical map, The International Journal of Control Automation and Systems Vol.3(No.1): 15-31.

Nienaber, G. \& Diekhans, N. (2006). Accuracy analysis of gps-based autoguidance systems, VDI BERICHTE Vol. 1958: 329.

Pein, C., Rőhrich, D., Skjodt, P. \& McClure, J. (2006). Combining gps and camera steering (lps) systems: in field applications and experiences, Proceeding of AgEng Bonn 2006, 64th VDI-MEG International Conference Agricultural Engineering, Bonn, p. 1958.

Perera, L. D. L., Wijesoma, W. S. \& Adams, M. D. (2006). The estimation theoretic sensor bias correction problem in map aided localization, The International Journal of Robotics Research Vol.25(No.7): 645-667.

Schwarz, G. (n.d.). Estimating the dimension of a model, The Annals of Statistics Vol.6(No.2): 461-464. 
Tessier, C., Berducat, M., Chapuis, R. \& Bonnet, S. (2007). Characterization of feature detection algorithms for a reliable vehicle localization, Proceeding of 6th IFAC Symposium on Intelligent Autonomous Vehicles, Toulouse, France.

Tessier, C., Debain, C., Chapuis, R. \& Chausse, F. (2006a). Active perception strategy for vehicle localization and guidance, Proceeding of the $2^{\text {th }}$ IEEE International IEEE Conference on Cybernetics $\mathcal{E}$ Intelligent Systems and Robotics, Automation $\mathcal{E}$ Mechatronics, Bangkok, Thailand, pp. 244-249.

Tessier, C., Debain, C., Chapuis, R. \& Chausse, F. (2006b). Fusion of active detections for outdoors vehicle guidance, Proceeding of the $9^{\text {th }}$ International IEEE Conference on Information Fusion, Florence, Italie, pp. 1914-1921.

Tessier, C., Debain, C., Chapuis, R. \& Chausse, F. (2007). A cognitive perception system for autonomous vehicles, Proceeding of COGIS'07 COGnitive systems with Interactive Sensors, Standford University California, USA.

Wu, N. \& Zhang, J. (2006). Factor-analysis based anomaly detection and clustering, Decision Support Systems Vol.42: 375-389. 


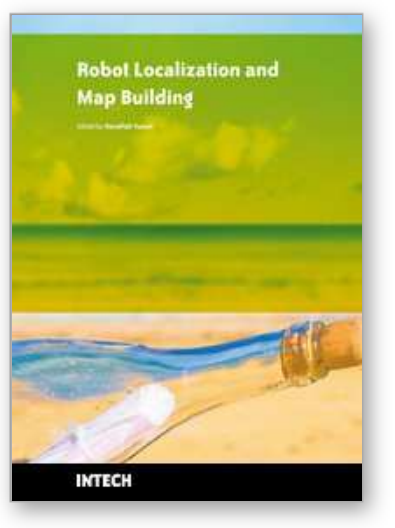

\author{
Robot Localization and Map Building \\ Edited by Hanafiah Yussof
}

ISBN 978-953-7619-83-1

Hard cover, 578 pages

Publisher InTech

Published online 01, March, 2010

Published in print edition March, 2010

Localization and mapping are the essence of successful navigation in mobile platform technology. Localization is a fundamental task in order to achieve high levels of autonomy in robot navigation and robustness in vehicle positioning. Robot localization and mapping is commonly related to cartography, combining science, technique and computation to build a trajectory map that reality can be modelled in ways that communicate spatial information effectively. This book describes comprehensive introduction, theories and applications related to localization, positioning and map building in mobile robot and autonomous vehicle platforms. It is organized in twenty seven chapters. Each chapter is rich with different degrees of details and approaches, supported by unique and actual resources that make it possible for readers to explore and learn the up to date knowledge in robot navigation technology. Understanding the theory and principles described in this book requires a multidisciplinary background of robotics, nonlinear system, sensor network, network engineering, computer science, physics, etc.

\title{
How to reference
}

In order to correctly reference this scholarly work, feel free to copy and paste the following:

Pierre Delmas, Christophe Debain, Roland Chapuis and Cedric Tessier (2010). Reliable Localization Systems Including GNSS Bias Correction, Robot Localization and Map Building, Hanafiah Yussof (Ed.), ISBN: 978-9537619-83-1, InTech, Available from: http://www.intechopen.com/books/robot-localization-and-mapbuilding/reliable-localization-systems-including-gnss-bias-correction

\section{INTECH}

open science | open minds

\section{InTech Europe}

University Campus STeP Ri

Slavka Krautzeka 83/A

51000 Rijeka, Croatia

Phone: +385 (51) 770447

Fax: +385 (51) 686166

www.intechopen.com

\section{InTech China}

Unit 405, Office Block, Hotel Equatorial Shanghai

No.65, Yan An Road (West), Shanghai, 200040, China

中国上海市延安西路65号上海国际贵都大饭店办公楼 405 单元

Phone: +86-21-62489820

Fax: $+86-21-62489821$ 
(C) 2010 The Author(s). Licensee IntechOpen. This chapter is distributed under the terms of the Creative Commons Attribution-NonCommercialShareAlike-3.0 License, which permits use, distribution and reproduction for non-commercial purposes, provided the original is properly cited and derivative works building on this content are distributed under the same license. 